\title{
Effects of GRAS substances on pharmacologic effects of drugs
}

\author{
Bert N. La Du, M.D., Ph.D. Ann Arbor, Mich. \\ Department of Pharmacology, University of Michigan Medical Center
}

First, let me explain what GRAS means. GRAS is an acronym for "generally recognized as safe." It refers to a large number of chemical compounds that the FDA considers to be safe when added to foods. The legal basis for GRAS status is rather complicated and goes back over many years to early federal regulations dealing with food additives. In 1958 the FDA solicited opinions from the scientific community about the usefulness and safety of all the substances then added to foods, and in 1959 and 1960 had lists published in the Federal Register of those that were considered GRAS and had not received adverse comments. ${ }^{1}$ No further testing of the safety of these initial GRAS compounds was required, but any new additions to the list were expected to be adequately evaluated for usefulness and safety. Over the years, GRAS status was granted to other compounds by the FDA officials by letters of approval, and the present number of GRAS substances is probably in excess of 700 compounds. There are also approximately 1,200 natural and synthetic flavoring agents added to foods which are generally recognized as safe by scientific experts but listed separately in the Code of Federal Regulations. $^{2}$

It may surprise pharmacologists familiar with the procedure for testing and approval of new drugs to learn that the presumed safety of so

Reprint requests to: Dr. Bert N. La Du, Department of Pharmacology, University of Michigan Medical Center, Ann Arbor, Mich. 48109 . many chemicals added to foods has been based upon common experience in usage and the lack of adverse information, rather than extensive toxicologic testing and knowledge of their biologic effects.

In 1969, the President directed the FDA to re-evaluate the safety status of all the GRAS substances. By 1972 a Select Committee was established to carry out this assignment under a contract from the FDA to the Federation of American Societies for Experimental Biology (FASEB). The Life Sciences Research Office of FASEB, directed by Dr. C. J. Carr, was the organizational base for this task, with a committee of about twelve members representative of the FASEB societies and other relevant scientific disciplines. As one of the members of the Select Committee on GRAS substances for nearly five years, I have had access to an unusual quantity of scientific literature related to GRAS substances, including that related to possible interactions with therapeutic agents. Committee members were provided with monographs on each substance summarizing the world's scientific literature from 1920 to 1970 , with supplemental material obtained through other sources such as Toxline and Medline from the National Library of Medicine. These resources include considerably more information on the GRAS substances than interactions with drugs, but the question of possible interactions of this type was specifically sought out in each literature survey. 
Table I. GRAS compounds-codes indicating purposes of use (Section 121.101, Code of Federal Regulations)

(a) GRAS substances because of common or general usage. Examples: salt, sugar, baking powder, pepper, monosodium glutamate

(d) GRAS substances for their intended use:

(1) Anticaking agents. Examples: tricalcium silicate, magnesium silicate

(2) Chemical preservatives. Examples: ascorbic acid, benzoic acid, butylated hydroxyanisole, butylated hydroxytoluene

(3) Emulsifying agents. Examples: cholic acid, glycocholic acid, mono- and diglycerides

(4) Nutrients and/or dietary supplements. Examples: amino acids, vitamins, minerals, sorbitol

(5) Sequestrants. Examples: calcium salts, citric acid, tartaric acid

(6) Stabilizers. Examples: plant gums, alginates, carrageenan

(7) Miscellaneous and/or general purpose food additives. Examples: acetic acid, beeswax, caffeine, gases, lecithin, lactic acid, adipic acid

(e) Spices, seasonings, natural extractives

(f) Trace minerals added to animal feeds

(g) Synthetic flavoring substances

(h) Substances migrating to food from paper and paperboard products used in food packaging. Examples: clay (kaolin), formic acid, alum, casein, ethyl acrylate, silicon dioxides, starch

(i) Substances migrating to food from cotton and cotton fabrics used in dry food packaging. Examples: lard, beef tallow, Japan wax, stearic acid

The Select Committee has been conducting a comprehensive review of the safety of each of the GRAS substances, writing reports on the current health aspects of these compounds with opinions and conclusions to the FDA concerning their continued use and status as GRAS compounds for the future. Obviously, my comments about the work of the Select Committee and the compounds we are reviewing are personal. They do not represent opinions or conclusions of the Committee, or those of the FDA.

Before discussing possible interactions between GRAS substances and drugs it is important to describe in more specific terms what types of compounds are included within the roughly 700 GRAS substances. There is no official, complete published list that can be referenced. The Select Committee has compiled a reasonably comprehensive list with the help of the FDA, and this will be included in a publication from the Select Committee in the near future. For this paper, it may be sufficient to indicate some of the general classes of compounds, according to use, with a few representative examples (Table I).

The purposes for adding these compounds to foods cover a broad spectrum-from anticaking agents (silicates in table salt) to those compounds which can migrate into foods from packaging materials (waxes and starch). The GRAS compounds vary widely in their chemical composition and nature. Some are highly purified, synthetic chemicals of well-defined structure; others are relatively crude, natural products, such as the plant gums. The detailed specifications for "food grade" GRAS substances with maximum levels of toxic metals and other specified contaminants permitted are generally to be found in the Food Chemicals Codex. ${ }^{3}$ For an appreciable number of GRAS substances, however, no specifications are given. Even when described in the Food Chemicals Codex, the tolerances permitted for many GRAS substances are sufficient to allow potentially toxic or undesirable impurities to be present. The specifications for the plant gums are particularly deficient in this respect. Some of the natural products used as seasoning or flavoring agents (garlic, dill, mustard, rue) are, by their nature, of variable composition and may contain ingredients that may be of concern, as safrole in nutmeg oil. Fortunately, most of the GRAS compounds added to foods or migrating into foods from packing materials are consumed in extremely small quantities, a few milligrams or less per day. Unless the diet is most unusual, or the components are highly toxic or potent, we are thus protected from a number of potential interactions with therapeutic agents which might otherwise occur. Hydrogenated fish oil is GRAS, and fish oils may be contaminated with insecticides and other residues that would be of concern if large amounts were eaten. The GRAS status of hydrogenated fish oil, however, applies only in regard to the amount migrating to food from cotton packag- 
ing material, not as a direct food ingredient. The limitation on use imposed by each of the GRAS categories (Table I) must be taken into account, not just the fact that the substance is GRAS, in considering possible interactions with therapeutic agents. Of course, some substances are GRAS for more than one purpose. Ascorbic acid is permitted as both a chemical preservative and as a nutrient. In calculating the total daily intake of the substances the multiple uses, if permitted, must be added.

An important point about the definition of GRAS substances should be mentioned. They are added to foods, of course, but from a legal standpoint, they are not "food additives." Food additives are substances added to foods within some specific tolerance level. GRAS substances can be added for the purpose intended with no specified limits other than those determined by standard manufacturing practice. There are a few exceptions, in which GRAS substances have food additive-like limitations. Caffeine is GRAS and is added to baked goods and to cola beverages; the concentration of caffeine in cola drinks cannot exceed $0.02 \%$ in the final product, taking into account the natural caffeine content of the cola extract. Pharmacologists and toxicologists familiar with the activity of caffeine as a stimulant and its other pharmacologic activities might anticipate that caffeine as a GRAS substances should lead to a number of interactions with other drugs. Limitation on the level of caffeine permitted in cola drinks reduces the probability for such interactions, unless large volumes are consumed.

The Select Committee has found the evaluation of the safety of GRAS substances to be a rather difficult assignment. Perhaps the most troublesome aspect of the review process has been the lack of adequate biologic and toxicologic information from the world literature on so many of these compounds. For many substances there is no adequate acute or chronic animal oral toxicity information, and relatively few GRAS substances have been studied in man to determine their metabolic fate and disposition. Oral administration is obviously the relevant route for evaluating the safety of the GRAS compounds. The Committee has often been in the position of having some parenteral toxicity data, but no information on the absorbability of the compound when given orally. The Select Committee has noted in its reports what additional information is most needed for each substance reviewed and has given an indication of priority ranking since to obtain all these scientific data would be an enormous undertaking and require many years of research.

A particularly difficult problem for the Committee has been the estimation of consumer exposure: what foods are consumed and how much of each are eaten by people of different ages and dietary habits? Data were supplied to the Select Committee by a National Research Council Subcommittee ${ }^{4}$ on the consumption of GRAS substances. Their survey is the most comprehensive of any available at present, but the estimates of average daily consumption of the various GRAS substances are regularly in excess of actual consumption. In some instances the NRC subcommittee estimates exceeded by 100 to 1,000 times the total amount of the substance known to be added to foods. The latter amounts could be calculated from poundage figures provided by producers of the compounds for food manufacturers. Safety evaluation requires a reasonable estimation of the amount consumed on the average, and the amounts special groups (babies, infants on special formulas, teenagers, etc.) might consume if their diet selection included foods containing the highest amounts of the GRAS substances. Another factor to consider is the current trend in the use of GRAS substances and the changing patterns in consumer eating habits. The Select Committee is expected to evaluate the health aspects of the GRAS substances as currently used, and as might reasonably be expected to be used in the future. For example, the NRC subcommittee figures indicate that the average person (over 2 years old) consumes over $30 \mathrm{gm}$ of sorbitol per day. Other estimates based on the quantity used in foods, or on production and import figures, are about one hundred times smaller. The higher figure is close to the level known to have a laxative effect in adults, and there would be reason for concern that people might be eating more sorbitol than they should; the lower, more realistic figure leads to the conclusion that the current intake, as an average 
figure, is not excessive. The uncertainties about the NRC subcommittee's figures have been recognized, and that group is now in the process of conducting a new survey and revising its methods for calculating consumption data.

\section{GRAS substances-drug interactions}

It may be disappointing to some, but I have found no serious instance of GRAS substancedrug interactions in all of the literature on GRAS compounds we have received which are not well known and generally recognized. Many inorganic salts and metal salts are GRAS, and there are numerous examples of one salt influencing the solubility and absorption of another, but this type of interaction needs no detailed discussion. Alginates, vegetable oils, phosphate salts, and other inorganic GRAS salts can influence the absorption of many trace metals as well as iron and calcium.

More specific interactions can be cited. Sulfite is known to cause the destruction of thiamine, but this has been recognized for many years. In fact, there is a specific federal regulation which prohibits the use of sulfites as chemical preservatives in foods that represent major sources of dietary thiamine. The use of sulfites in foods presents a special problem in calculating the amount present in foods as eaten. Most of the sulfite is used during food processing steps, and much of it is lost before consumption.

Another possible interaction with drugs can be proposed on the basis of animal studies with the GRAS compounds: BHA (butylated hydroxyanisole) and BHT (butylated hydroxytoluene). Both compounds are added to foods as chemical preservatives but both are regulated GRAS and can be added up to $0.02 \%$ of the fat content of the food, such as potato chips. These antioxidants are effective inducers of liver microsomal enzymes and cause hepatic hypertrophy. If enough were eaten, the compounds might induce more rapid metabolism of many drugs in the liver. The question remains unanswered as to whether the intake of these substances (within the tolerance limit specified) in man is sufficient to produce a significant inductive effect on drug metabolism, and perhaps on steroid hormone metabolism in the liver.
BHT is more potent than BHA as an inducer in the animals studied, but more human studies are needed.

There is a special type of allergic hypersensitivity associated with some of the plant gums which should be mentioned. Gum acacia, tragacanth, and karaya are the ones most frequently involved, and there appears to be some cross-sensitivity among these gums. The plant gums are used as stabilizers and thickening agents in a variety of foods so that it is difficult to avoid them if an allergic reaction develops. Some of these gums, particularly gum acacia, are also used in a number of pharmaceutical preparations. A hypersensitive person might appear to be allergic to a drug, but really be reacting to the plant gum in the pharmaceutical preparation.

One can imagine the possibility of competitive interactions between drugs and GRAS substances in metabolic reactions, such an conjugation with glycine. Both aspirin and benzoic acid are converted to glycine conjugates in the liver. It is extremely unlikely, however, that enough benzoic acid would ever be present in the food to interfere with salicylic acid conjugation.

In general, the low consumption of most GRAS substances precludes the interactions that would be expected if large amounts of these same compounds were ingested. Of course, recent advances in our understanding of the complex systems involved in drug metabolism open additional opportunities for interactions and modulation of drug metabolism by GRAS compounds. The lack of new and unexpected examples of interactions in the GRAS compound literature may be more because of a lack of knowledge than an analysis based on adequate scientific evidence. There is certainly a need for continued alertness to such possibilities by clinical pharmacologists.

\section{References}

1. General Services Administration, Office of the Federal Register, Code of Federal Regulations, Section 121.101, in Code of Federal Regulations. Title 21, Food and Drugs. Parts 10 to 199 rev., Washington, D. C., 1976, U. S. Government Printing Office.

2. General Services Administration, Office of the Federal Register, Code of Federal Regulations, 
Section 121.1163 and 121.1164, in Code of Federal Regulations. Title 21. Food and Drugs. Parts 10 to $199 \mathrm{rev}$, , Washington, D. C., 1976, U. S. Government Printing Office.

3. National Research Council, Committee on Food Protection: Food Chemicals Codex, ed 2, Washington, D. C., 1972, National Academy of Sciences.

4. National Research Council, Committee on Food Protection, Subcommittee on Review of GRAS List (Phase II): A comprehensive survey of industry on the use of food chemicals generally recognized as safe (GRAS), Washington, D. C., 1972, National Academy of Sciences.

\section{Discussion}

Dr. Vesell: Hershey, $\mathrm{Pa}$.: I have heard estimates by people testifying before Congress that although it may be minute amounts in any one product, that humans consume approximately three pounds a year of all the GRAS and related substances.

But more important, really, is an anecdotal study that $I$ heard about in which a physician, or actually several physicians, have started putting hyperkinetic children on diets that are as free of these additives as possible and state rather dogmatically that these children improved rather markedly.

Dr. La Du: I have a reprint with me called Report to the Nutrition Foundation by the National Advisory Committee on Hyperkinesis and Food Additives.

A national study has been undertaken to answer this question about hyperkinetic children and food additives. Dr. B. Feingold in California has claimed that the hyperactivity in children is due to these food additives.

However, his main target is the food coloring materials, Red No. 2, among others.

Our Select Committee is not reviewing these additives. I don't know much about them, but there have been enough people with similar claims and a national committee was selected, which looked over the information and decided that the only solution was to have the proper kind of scientific study, and this is now under way.

It's hard for me to imagine that small amounts of these substances can interfere with metabolism and affect behavior so dramati- cally. There are enough reports from doctors who have treated children and say that they have been cleared of their hyperkinesis by the exclusion of these food additives so it is worth studying carefully.

Dr. Melmon, San Francisco, Calif.: Your Select Committee is expected to study the safety or toxicity of substances which have been classified as being generally recognized as safe.

Has that committee reviewed the reasons or the rationale for including or excluding substances from the GRAS list? How does the GRAS list differ from food additives and dyes? Are the others being evaluated?

Dr. La Du: We were asked by the FDA to review just the GRAS substances. Another committee like ours is going to review the flavoring agents and another committee like ours presumably will review the food coloring agents.

Questions came up very early in our Committee about what we were to do about GRAS substances where there was very little information. In other words, is the safest compound the one you know the least about? As a scientific committee, we would like some minimal standards for GRAS approval, but we realized that if we insisted on standards that would make sense in terms of drug safety, nothing would be approved as GRAS. There are compounds that we have refused to review, saying that there just wasn't enough information. We also have made recommendations about what ought to be done further with many GRAS substances and this is included in our reports. We've tried to be selective about recommendations and have given reasonable priorities about what is needed most to do.

This is the first time that these compounds have ever been reviewed in this way. Even the World Health Organization didn't have the world literature collected, assimilated, and organized for them.

Dr. Murphy, Boston, Mass.: You mentioned the gums and the evidence suggesting there were allergic reactions in some people associated with these. What does your Committee feel is the solution to this kind of a hypersensitivity?

Dr. La Du: We feel that one step is to define 
better standards of purity and composition. Present standards allow proteinaceous material in the gums which may be important in determining allergic reactions. There is the general problem with many of these natural GRAS substances. Better standards for food grade materials would help establish whether the allergic reactions are due to impurities or to gum material, itself.

The other thing that would help is to identify which gum is present on the label of the product. Simply indicating vegetable gum or plant gum doesn't help the allergic consumer. Labeling contents, however, is a complicated problem for food products and the FDA.

Dr. Murphy: What you are saying about gums could apply to vegetable oils because the same situation holds. The label may say pure vegetable oil, but the mixture changes from season to season, year to year, depending on whether the crop of peanuts was good that year or not. These, oftentimes, are contaminated with protein from the vegetables, and people know that they are allergic to certain proteins but can't tell whether they are present in the oils or not.

Dr. La Du: We have suggested this problem might be solved by labeling, but the FDA may find a better solution.

Dr. Murphy: Have you noticed, in your committee, as I experienced on a similar committee related to the flavors, that an allergic response to a food additive is of little concern because, theoretically, it occurs at low frequency; yet, when you ask what frequency, it may be as much as one percent. As you know, this is a pretty high frequency.
Dr. La Du: I think our Committee is dealing regularly with problems like this. Averaging intake for the total population is not very helpful, because we know the "average" doesn't apply to select groups within the population.

High iron intake, for example, may be a hazard to a subpopulation that has a disturbance in the regulation of iron absorption, leading to iron accumulation in the liver if they take extra iron in their food. Probably most people can tolerate this load of iron, but is it good for the public to have the daily iron requirement present in about six different food products?

Dr. Murphy: Isn't there also the problem of knowing exactly what you are getting?

If the standards for food grade GRAS substances are so broad, they can include other things. Even if you were able to single out representative populations to test unusual reactions, you'd still not be giving something that would be a uniform and representative GRAS substance.

Dr. La Du: Yes, the food grade specifications could certainly be improved. For example, talc-coated rice is still available, but there is no definition of food grade talc. Talc can contain asbestos fibers; but you get into the difficult question, then, of whether oral asbestos fibers are a hazard or not. Most of the food grade specifications were developed years ago based on analytic methods of that period. Newer methods of analysis would be worthwhile in assuring quality of the substances added to foods and help clear up some of the uncertainty about the importance of contaminants in explaining unusual or allergic reactions to some of the GRAS substances. 